\title{
Associations of total and type-specific physical activity with mortality in chronic obstructive pulmonary disease: a population-based cohort study
}

Sonia Wing Mei Cheng ${ }^{1 *}$, Zoe McKeough ${ }^{1}$, Jennifer Alison ${ }^{1}$, Sarah Dennis ${ }^{1,2}$, Mark Hamer $^{3}$ and Emmanuel Stamatakis ${ }^{4}$

\begin{abstract}
Background: Regular physical activity is recommended for all people with chronic obstructive pulmonary disease (COPD), but the dose of physical activity required to gain mortality benefit in this population is not yet known. This aim of this study was to examine the associations of total and type-specific physical activity with mortality risk in people with COPD.

Methods: People with COPD aged $\geq 40$ years were identified from the 1997 Health Survey for England and the 1998 and 2003 Scottish Health Survey cohorts. Self-reported total physical activity, moderate-vigorous intensity physical activity (MVPA), walking, domestic physical activity, and sport/exercise were assessed at baseline. Cox proportional hazards models were used to examine the associations between physical activity and mortality risk.

Results: Two thousand three hundred ninety-eight participants with COPD were included in the analysis and followed up for a mean 8.5 (SD 3.9) years. For both total physical activity and MVPA, we observed doseresponse associations with all-cause and cardiovascular disease (CVD) mortality risk, and with respiratory mortality risk to a lesser extent. Compared to those who reported no physical activity, participants who met the physical activity guidelines demonstrated the greatest reductions in all-cause ( $\mathrm{HR} 0.56,95 \% \mathrm{Cl} 0.45-0.69$ ), CVD (HR 0.48, 95\% Cl 0.32-0.71) and respiratory mortality risk (HR 0.40,95\% Cl 0.24-0.67). Participants who reported a level of physical activity of at least half the dosage recommended by the guidelines also had a reduced risk of all-cause (HR 0.75, 95\% Cl 0.56-1.00) and CVD mortality (HR 0.48, 95\% Cl 0.26-0.88). Doseresponse associations with mortality risk were demonstrated for walking and sport/exercise, but not domestic physical activity.
\end{abstract}

Conclusions: We found a dose-response association between physical activity and all-cause and CVD mortality risk in people with COPD, with protective effects appearing at levels considerably lower than the general physical activity recommendations. People with COPD may benefit from engagement in low levels of physical activity, particularly walking and structured exercise.

Keywords: Chronic obstructive pulmonary disease, Physical activity, Mortality, Epidemiology

\footnotetext{
* Correspondence: sonia.cheng@sydney.edu.au

${ }^{1}$ Discipline of Physiotherapy, The University of Sydney, Sydney, Australia

Full list of author information is available at the end of the article
} 


\section{Background}

Compared to their healthy peers, people with chronic obstructive pulmonary disease (COPD) engage in a lower intensity of daily physical activity (PA), and spend significantly more time sitting and lying down $[1,2]$. This has been attributed to disease-specific limitations such as dyspnoea, fatigue and reduced exercise tolerance, which reduce the capacity of people with COPD to engage in moderate-vigorous intensity physical activity (MVPA) [3]. Previous cohort studies have consistently shown that low levels of PA are associated with an increased risk of all-cause and respiratory mortality in people with COPD, as well as an increased risk of COPD exacerbations, irrespective of the degree of airflow obstruction [4]. Low levels of PA are also associated with an increased risk of hospitalisation, re-hospitalisation after an acute exacerbation, and longer hospital length of stay $[4,5]$.

Although regular PA is recommended for all patients with COPD [6], there is a lack of high-quality evidence on which to base specific PA recommendations for this population, in particular the dose of PA required to gain mortality benefit. A Spanish cohort study of 611 people with COPD found that those who "took walks regularly for $>8 \mathrm{~km}$, no less than 5 days a week, or practised sports" had a lower mortality risk compared to those who "don't leave the house and life is limited to the bed or armchair, or to doing some domestic chores" (risk ratio (RR) 0.38, 95\% confidence interval (CI) 0.11-1.29) [7]. Another cohort study of 173 people with moderate to very severe COPD, which used accelerometry to measure PA levels, found that every ten vector-magnitude unit (VMU) increase in daily PA was associated with a $14 \%$ reduction in mortality risk [8]. Such studies are limited by the use of small non-representative samples of people with COPD to examine the association between PA and mortality risk, and by arbitrary definitions of what constitutes "low" and "high" levels of PA. Many different questionnaires and activity monitors have been used to assess PA in people with COPD, and the optimal mode of reporting (e.g., energy expenditure, number of steps/day) is not yet known [4, 9].

The considerable heterogeneity in the way that PA has been classified and reported in previous studies has made it difficult to develop PA guidelines specific to people with COPD, and subsequently clinicians must rely on the PA guidelines for the general population to inform clinical recommendations [10]. It is unknown whether specific types of PA, such as walking and domestic PA, are associated with reductions in mortality risk. Exploring types of PA that are relevant to functionally limited populations such COPD, and the dose of PA required to gain mortality benefit, is necessary in order to guide the prescription of PA in clinical practice and to inform interventions which aim to increase PA.

The aim of this study was to examine the associations between total and type-specific PA and mortality risk in a series of representative cohorts of adults with COPD, specifically:

1. The dose-response association between total PA and the risk of all-cause, cardiovascular disease (CVD), and respiratory mortality within the context of current public health recommendations; and

2. The dose-response association between specific types of PA and the risk of all-cause, CVD, and respiratory mortality.

\section{Methods}

\section{Study design}

The Health Survey for England (HSE) and Scottish Health Survey (SHS) cohorts are general population-based health examinations of individuals aged $\geq 16$ years living in households in England and Scotland respectively. Participants were selected using a multistage stratified probability design, based on geographical location, to provide a nationally representative sample of the population in each constituent country. Further details on the HSE/SHS sampling methods can be found elsewhere $[11,12]$.

Participants for the present analysis were drawn from the HSE and SHS survey cohorts with available data on lung function and PA (HSE 1997 and SHS 1998, 2003), and were linked prospectively to National Health Service mortality data. The surveys consisted of two household visits: an interviewer visit, to collect information on physical and mental health and health behaviours, followed by a nurse visit to obtain physiological measures. Response rates for the interviewer visit ranged from 76 to $83 \%$, and 31 to $44 \%$ for the subsequent nurse visit. Ethics approvals for the HSE and SHS were obtained from the London Research Ethics Council and Scotland Local Research Ethics Councils respectively. Participants gave written informed consent.

\section{Participants}

There were two eligibility criteria to identify participants with COPD: (1) age $\geq 40$ years, and (2) meeting the Global Initiative for Chronic Obstructive Lung Disease (GOLD) spirometric criteria for COPD (forced expiratory volume in one second $\left(\mathrm{FEV}_{1}\right) /$ forced vital capacity $(\mathrm{FVC})$ ratio of <0.7) [3]. Participants were followed-up to the time of death or, if there was no record of an event, data were censored at February 15, 2011 (HSE) and at December 31, 2009 (SHS). 


\section{Measurements}

\section{Participant characteristics}

Interviewers used computer-assisted interviewing modules to collect information on participants' demographic and socioeconomic characteristics, self-reported health status, and other health behaviours (e.g., smoking, alcohol consumption).

\section{Lung function}

Spirometry was performed using a Vitalograph ${ }^{\oplus}$ Escort spirometer, which was calibrated prior to each participant's use. Participants who normally required bronchodilator medication prior to strenuous exercise were allowed to take their usual medication before spirometry was performed. No additional bronchodilators were given by the survey nurses. Participants performed at least one practice attempt, followed by five attempts or until they were deemed too tired to continue. The best of the five attempts (excluding any technically unsatisfactory attempts, e.g., coughing during the blow, air leak around the mouthpiece) was used as the final result. The full spirometry protocol has been published elsewhere [13].

\section{Physical activity}

The Physical Activity and Sedentary Behaviour Assessment Questionnaire (PASBAQ) was used to collect data on physical activity. Participants reported the frequency (number of days in the last 4 weeks) and duration (of an average episode) of participation in four domains of PA: (1) "light" (e.g. general tidying) and "heavy" (e.g. spring cleaning) domestic activity; (2) "light" and "heavy" manual work, gardening and "doit-yourself" activities; (3) light-intensity (slow/average pace) and moderate-intensity (fairly brisk/fast pace) walking; and (4) sport/exercise. Intensity of sport/exercise was determined by whether the activity had made participants "out of breath or sweaty", and by the nature of the activity as indexed in the metabolic equivalent (MET) compendium [14]. The PASBAQ has demonstrated satisfactory validity when compared against accelerometry data in a representative sample of British adults; Spearman's correlation coefficients were $0.20 \quad(95 \%$ confidence interval (CI) $0.25-0.35$ ) in men and 0.30 (95\% CI 0.15-0.26) in women [15]. Participation in PA was then calculated in MET-hours/week by multiplying the volume of activity (frequency $\mathrm{x}$ duration) by the intensity of the activity in METs [14].

Five PA variables were derived for the analyses: METhours/week of total non-occupational PA, walking, domestic PA, and sport/exercise, and minutes/week of MVPA. Total PA and MVPA were converted into four categories based on adherence to the general PA recommendations (i.e., at least $150 \mathrm{~min} /$ week of moderate-intensity aerobic PA (MPA), or at least $75 \mathrm{~min} /$ week of vigorous-intensity aerobic PA (VPA), or an equivalent combination of MVPA) [10]. This recommendation is equivalent to 7.5 METhours/week of PA [16]. Participants were classified as 'Inactive' (0 MET-hours/week of total PA, 0 min/week of MVPA), 'Insufficiently Active (Low)' (< 3.75 MET-hours/ week of total PA, $<75 \mathrm{~min} /$ week of MPA or equivalent combination of MVPA), 'Insufficiently Active (High)' (3.75 to <7.5 MET-hours/week of total PA, 75 to < $150 \mathrm{~min} /$ week of MPA or equivalent combination of MVPA), or 'Sufficiently Active' ( $\geq 7.5$ MET-hours/week of PA, $\geq 150 \mathrm{~min} /$ week of MPA or equivalent combination of MVPA). Participants who reported no PA were specified as the referent group. This method of classifying PA has been used in other cohort studies of people with COPD [5].

The walking, domestic PA, and sport/exercise variables were converted into three categories. The referent group was specified as no participation in walking, domestic PA, or sport/exercise respectively ('No PA'). For each type of PA, the remaining participants were divided into one of two groups: below the median level of activity of the cohort ('Low PA'), or equal to or above the median level of activity of the cohort ('High PA'). The medians were 5.25 METhours/week for walking, 5.70 MET-hours/week for domestic PA, and 8.00 MET-hours/week for sport/ exercise. Previous studies have used this approach to distribute participants evenly into each category and ensure that the Cox proportional hazards assumption is met [17].

\section{Mortality}

The study outcomes were all-cause mortality, CVD mortality, and respiratory mortality. The primary cause of death was identified from the death certificate and from any additional information provided by the certifying physician, and was recorded according to the International Classification of Diseases (ICD) Ninth and Tenth Revisions. CVD codes were 390 to 459 (ICD-9) and I01 to I99 (ICD-10), and respiratory codes 460 to 519 (ICD-9) and J00 to J99 (ICD-10).

\section{Covariates}

Potential confounders were identified a priori from a review of current literature $[5,16,18]$ and the most parsimonious selection of variables that achieved the greatest control of confounding was used. The following covariates were included in the analyses: age, sex, ethnicity (Caucasian, non-Caucasian), severity of COPD (mild, moderate, severe, or very severe according to the GOLD stages [3]), history of CVD at baseline, history of cancer at baseline, history of diabetes at baseline, self-reported longstanding illness, body mass index (BMI), smoking status (current smoker, ex-smoker, never smoked), age finished full-time education ( $<14$ years, 15-18 years, 
$\geq 19$ years), and alcohol consumption (does not drink, less than once a week, 1-4 times/week, $\geq 5$ times/week).

\section{Statistical analyses}

Differences in the distribution of baseline characteristics were examined using chi-square tests for categorical variables and analysis of variance (ANOVA) for continuous variables. Cox proportional hazards regression models were used to compute hazard ratios (HR) with 95\% CIs and compare mortality risk across the categories of PA. The proportional hazards assumption was examined by comparing the cumulative hazard plots of the five PA variables. No appreciable violations were noted. Covariates were added to the model in three stages: age, sex and ethnicity (Model 1); plus severity of COPD, history of CVD, history of cancer, history of diabetes, self-reported longstanding illness, and BMI (Model 2); plus smoking status, age finished full time education, and alcohol consumption (Model 3). Analyses of walking, domestic PA and sport/ exercise were also mutually adjusted for the other types of PA. Participants with missing data for mortality $(n=243)$ or for any of the covariates $(n=490)$ were excluded from the analyses.

Two sensitivity analyses were performed. To minimise the potential for reverse causality in the main analysis (i.e., that participants had an increased risk of mortality because of pre-existing medical conditions rather than their PA levels), the first excluded those who had died within the first 12 months of follow-up $(n=49)$, and those with existing CVD $(n=339)$, cancer $(n=135)$, and diabetes $(n=123)$ at baseline. Additional sensitivity analyses were conducted to exclude those who had died within the first 2 years of follow-up $(n=124)$ and within the first 3 years of follow-up $(n=196)$. As no appreciable differences were found, these participants were retained in the main analyses. To assess for misclassification of a diagnosis of COPD, the second excluded participants who had never smoked $(n=664)$, those with a history of asthma $(n=243)$, and those who reported a respiratory infection in the 3 weeks prior to the first household visit $(n=353)$. All statistical tests were two-sided, with a $p$-value $<0.05$ considered statistically significant. All analyses were performed using SPSS Version 22.0 (IBM Corp, USA).

\section{Results}

Of the 19,000 participants with available data on lung function and PA, 2398 participants met the eligibility criteria for COPD and were included in the analysis. The mean age of the sample was 62.6 (SD 11.5) years and $52 \%$ were men. There was a high prevalence of COPD of mild (42.7\%) and moderate (37.5\%) severity, and of never-smokers (27.9\%). Baseline characteristics, grouped according to the degree of adherence to the general PA recommendations, are displayed in Table 1. Participants who reported no PA were older, had COPD of greater severity, had more medical comorbidities, and had a lower level of education $(p<0.001)$. Mortality data were available for 2155 participants. Over a mean 8.5 (3.9) years follow-up, there were 571 deaths (26\%) of which 172 were CVD-related deaths and 105 were respiratoryrelated deaths, corresponding to a total of 18,222 person years.

The associations of MET-hours/week of total PA and minutes/week of MVPA with mortality risk are shown in Table 2. Dose-response associations with all-cause, CVD and respiratory mortality risk were demonstrated for total PA and MVPA. Participants in the 'Insufficiently Active (High)' group for total PA had a reduced risk of all-cause and CVD mortality compared to those who reported no PA, but no significant mortality risk reductions were observed in the 'Insufficiently Active (Low)' group for total PA (Fig. 1). Conversely, participants in the 'Insufficiently Active (Low)' group for MVPA had a reduced risk of all-cause mortality compared to those who reported no MVPA (Fig. 2). These dose-response associations were also observed in the sensitivity analyses (Additional file 1: Tables S1 and S2).

The associations of MET-hours/week of walking, domestic PA, and sport/exercise with mortality risk are shown in Table 3. Dose-response associations with allcause, CVD and respiratory mortality risk were demonstrated for walking, with no significant mortality risk reductions observed in the 'low' walking group. There was a dose-response association between sport/exercise and all-cause mortality risk, but not CVD or respiratory mortality risk. The few number of CVD and respiratory deaths in the 'low' and 'high' categories of sport/exercise have likely contributed to this result. Domestic PA of any level was not associated with reductions in mortality risk. Similar results were demonstrated in the sensitivity analyses (Additional file 1: Tables S3 and S4).

\section{Discussion}

This study of 2398 participants who met the GOLD spirometric criteria for COPD provides a detailed analysis of the dose-response associations between PA and mortality risk within the context of current public health recommendations, and for specific types of PA. The use of established thresholds to examine PA in both METhours/week and minutes/week provides clinicians with a meaningful reference standard to guide prescription of $\mathrm{PA}$, and enables future studies to make direct comparisons to the magnitude of mortality risk described in this sample. This is the first population-based cohort study of people with COPD to examine whether specific types of PA confer mortality benefit, which has high clinical 
Table 1 Baseline characteristics of 2398 participants with COPD recruited from the Healthy Survey of England (1997) and Scottish Health Survey $(1998,2003)$

\begin{tabular}{|c|c|c|c|c|c|c|}
\hline Variable & $\begin{array}{l}\text { All participants } \\
(n=2398)\end{array}$ & $\begin{array}{l}\text { Inactive }^{d} \\
(n=489)\end{array}$ & $\begin{array}{l}\text { Insufficiently } \\
\text { Active } \\
\text { (Low) }^{\text {e }} \\
(n=386)\end{array}$ & $\begin{array}{l}\text { Insufficiently } \\
\text { Active }(H i g h)^{f} \\
(n=225)\end{array}$ & $\begin{array}{l}\text { Sufficiently } \\
\text { Active }^{g} \\
(n=1268)\end{array}$ & $p$-value \\
\hline Mean age, years & $62.6(11.5)$ & $67.9(11.6)$ & $63.8(10.9)$ & $62.5(11.3)$ & $60.1(11.0)$ & $<0.001$ \\
\hline Men, \% & 52.1 & 54.6 & 47.9 & 47.1 & 53.5 & 0.06 \\
\hline Caucasian, \% & 98.9 & 98.8 & 98.7 & 99.2 & 99.0 & 0.92 \\
\hline $\mathrm{FEV}_{1} \%$ predicted $^{\mathrm{a}}, \%$ & & & & & & $<0.001$ \\
\hline GOLD I: $\geq 80 \%$ pred. & 42.7 & 26.0 & 37.8 & 41.1 & 49.7 & \\
\hline GOLD II: $50 \% \geq \mathrm{FEV}_{1} \%<80 \%$ pred. & 37.5 & 40.5 & 41.2 & 39.0 & 35.2 & \\
\hline GOLD III: $30 \% \geq$ FEV $_{1} \%<50 \%$ pred. & 14.5 & 25.2 & 15.4 & 15.8 & 10.6 & \\
\hline GOLD IV: FEV $<30 \%$ pred. & 5.3 & 8.3 & 5.6 & 4.1 & 4.5 & \\
\hline History of $C V D^{b}, \%$ & 14.1 & 28.4 & 18.7 & 11.8 & 7.7 & $<0.001$ \\
\hline History of cancer, \% & 5.6 & 6.1 & 6.0 & 5.1 & 5.4 & 0.91 \\
\hline History of diabetes ${ }^{c}, \%$ & 5.1 & 10.0 & 7.3 & 4.7 & 2.7 & $<0.001$ \\
\hline Self-reported longstanding illness, \% & 62.2 & 85.5 & 69.2 & 61.2 & 51.3 & $<0.001$ \\
\hline Self-reported asthma, \% & 10.1 & 13.9 & 10.6 & 9.8 & 8.6 & 0.01 \\
\hline Mean BMl, kg/m² & $26.6(4.7)$ & $27.3(5.5)$ & $27.1(4.9)$ & $26.6(5.0)$ & $26.3(4.3)$ & 0.001 \\
\hline Smoking status, $\%$ & & & & & & $<0.001$ \\
\hline Current & 37.2 & 42.7 & 38.6 & 38.6 & 34.4 & \\
\hline Ex & 34.9 & 37.1 & 33.2 & 36.2 & 34.3 & \\
\hline Never & 27.9 & 20.2 & 28.2 & 25.2 & 31.3 & \\
\hline Aged finished full-time education, \% & & & & & & $<0.001$ \\
\hline$\leq 14$ years & 28.7 & 45.7 & 32.6 & 27.1 & 21.2 & \\
\hline $15-18$ years & 59.4 & 48.2 & 57.8 & 61.2 & 63.8 & \\
\hline 19 years and older & 12.0 & 6.1 & 9.6 & 11.8 & 15.0 & \\
\hline Alcohol consumption, \% & & & & & & $<0.001$ \\
\hline Does not drink & 13.1 & 22.5 & 13.5 & 11.4 & 9.6 & \\
\hline Less than once per week & 26.9 & 27.4 & 31.9 & 30.2 & 24.6 & \\
\hline 1-4 times/week & 37.4 & 29.2 & 35.0 & 35.7 & 41.6 & \\
\hline$\geq 5$ times/week & 22.6 & 20.9 & 19.7 & 22.7 & 24.1 & \\
\hline
\end{tabular}

Values are means (SD) unless otherwise stated

FEV ${ }_{1}$ forced expiratory volume in 1 second, CVD cardiovascular disease, BMI body mass index

${ }^{a}$ COPD severity was classified according to Global Initiative for Lung Disease (GOLD) stages

${ }^{\mathrm{b}} \mathrm{A}$ history of cardiovascular disease was defined as self-reported or doctor-diagnosed angina, heart attack or stroke

${ }^{\mathrm{C}} \mathrm{A}$ history of diabetes was defined as self-reported or doctor-diagnosed diabetes, and glycated haemoglobin $(\mathrm{HBa} 1 \mathrm{C}) \geq 6.5 \%$

dParticipants who reported no physical activity during the week

eParticipants who reported $<3.75$ MET-hours/week of physical activity

fParticipants who reported between 3.75 and $<7.5$ MET-hours/week of physical activity

${ }^{9}$ Participants who reported $\geq 7.5$ MET-hours/week of physical activity as per current public health recommendations

relevance when developing interventions to increase PA in people with COPD.

Dose-response associations with mortality risk were demonstrated for total PA, MVPA, walking and sport/exercise, but not domestic PA. A previous Danish population-based cohort study of people with COPD, which compared subjects who reported low, moderate, or high levels of PA to subjects who reported a very low level of PA, showed that some level of regular PA was associated with a lower risk of all-cause and respiratory mortality [18]. In our study, reductions in mortality risk of the greatest magnitude were observed in participants who adhered to the PA guidelines. Compared to those who reported no PA, participants who were 'Sufficiently Active' showed a $45 \%$ reduction in allcause mortality risk, a $50-55 \%$ reduction in CVD mortality risk, and a $55-60 \%$ reduction in respiratory mortality risk.

Although there is added benefit in adhering to the PA guidelines, meeting such recommendations is often difficult for people with symptomatic COPD. This study showed that participants who did not meet the guidelines 


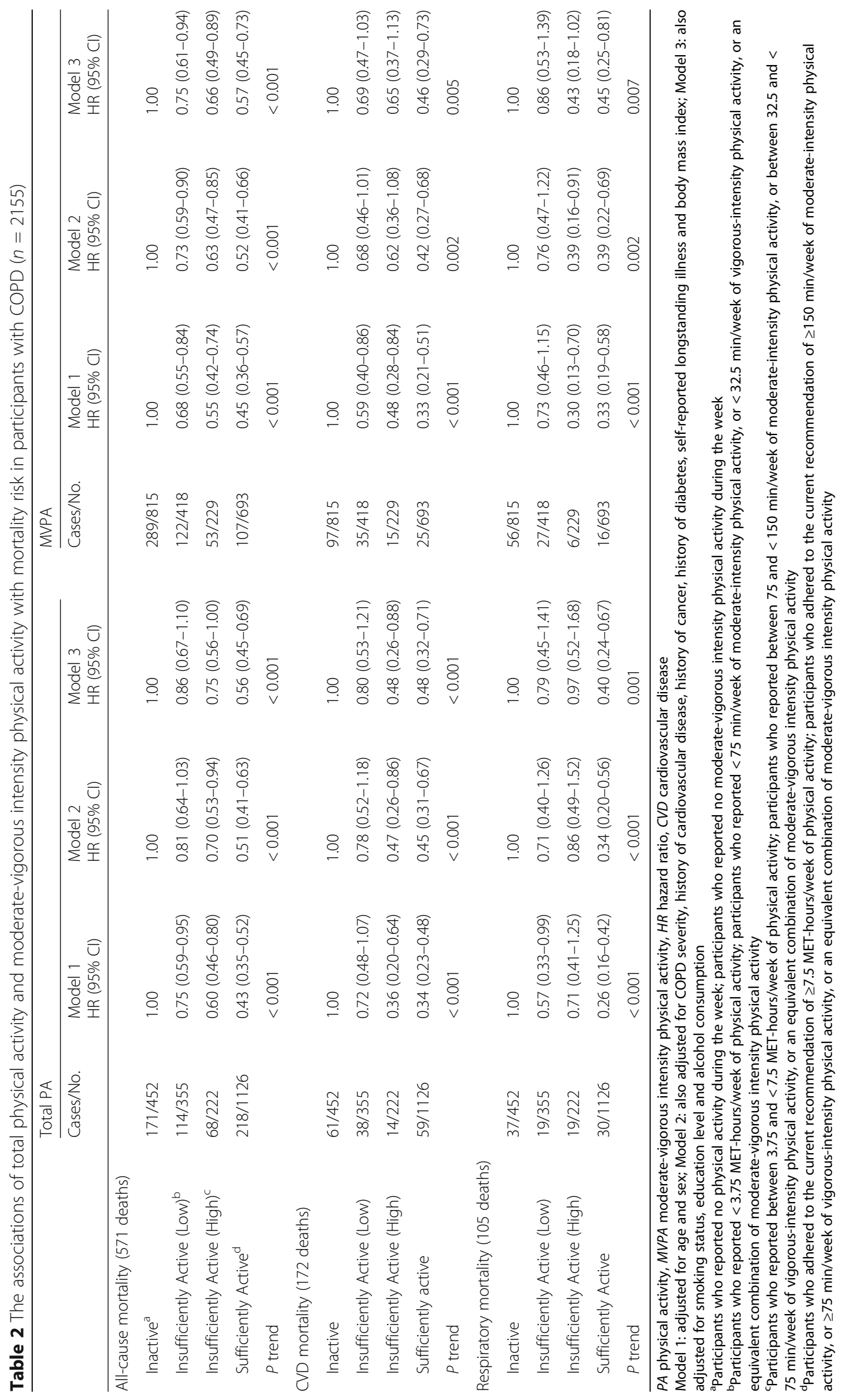




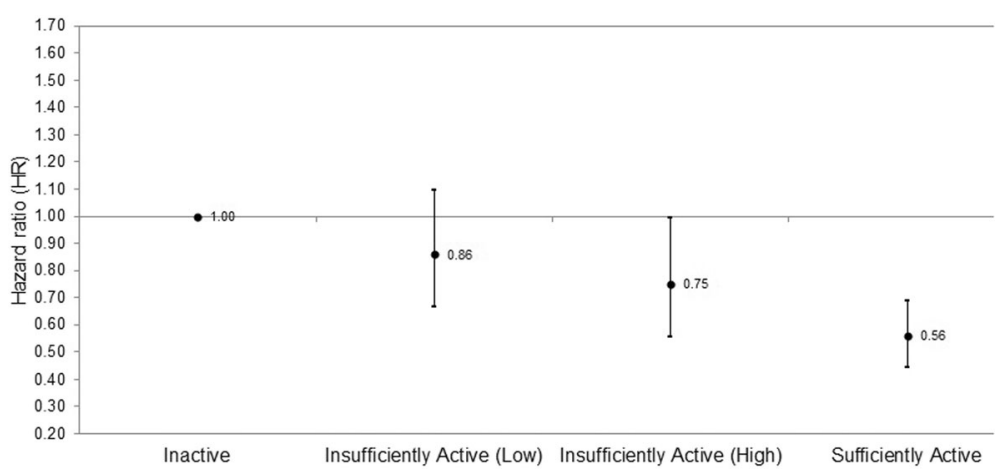

Fig. 1 The association of total physical activity with all-cause mortality risk in participants with COPD $(n=2155)$. The figure shows the dose-response association of total physical activity (PA) with all-cause mortality risk based on adherence to the general PA recommendations. Participants were classified as 'Inactive' (0 MET-hours/week of total PA), 'Insufficiently Active (Low)' (<3.75 MET-hours/week of total PA), 'Insufficiently Active (High)' (3.75 to $<7.5$ MET-hours/week of total PA), or 'Sufficiently Active' ( $\geq 7.5$ MET-hours/week of PA)

but reported at least 3.75 MET-hours/week of total PA, or reported some level of MVPA, still had significant reductions in mortality risk. After taking the sensitivity analyses into consideration, which yielded similar mortality risk reductions in participants without existing CVD, cancer and diabetes, these results suggest that the dose of PA required to gain mortality benefit in COPD may be lower than is currently estimated. This is consistent with previous observations that relatively low levels of PA have beneficial effects on mortality risk in people with COPD. It has been suggested that as little as $2 \mathrm{~h} /$ week of walking or cycling is associated with a $30-40 \%$ risk reduction in respiratory mortality and COPD hospitalisation [18].

In both the main and sensitivity analyses, participants in the 'Insufficiently Active (Low)' group for MVPA had a significant reduction in all-cause mortality risk (HR 0.75 , 95\% CI 0.61-0.94). However, no significant reductions in all-cause mortality risk were observed in participants who reported < 3.75 MET-hours/week of total PA (HR 0.86, 95\% CI 0.67-1.10). This subtle difference between the two equivalent measures of PA is likely due to error in the calculation of MET-hours/week of total PA, in which the intensity of PA is approximated according to the nature of the activity as indexed in the MET compendium. Whether higher intensity PA has beneficial effects on mortality risk independent of the volume of activity is not yet known in people with COPD. A Spanish cohort study demonstrated a $20 \%$ reduction in the risk of COPD hospitalisation for every additional 1000 daily steps at low average intensity, but no change in hospitalisation risk with additional steps at high average intensity [19]. Conversely, higher intensity PA was associated with a lower prevalence of metabolic syndrome in 223 subjects with COPD drawn from the National Health and Nutrition Examination Survey 20032006 dataset [20]. Considering the innate challenges of

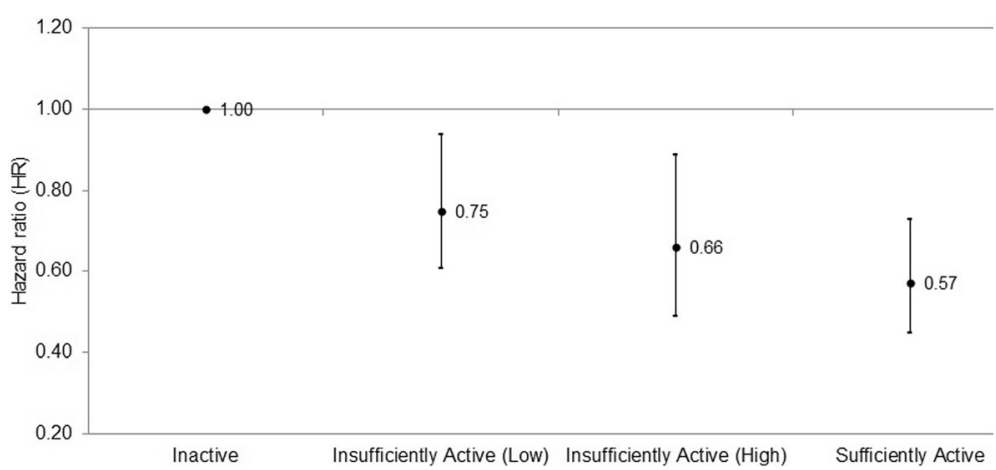

Fig. 2 The association of moderate-vigorous intensity physical activity with all-cause mortality risk in participants with COPD $(n=2155)$. The figure shows the dose-response association of moderate-vigorous physical activity (MVPA) with all-cause mortality risk based on adherence to the general physical activity (PA) recommendations. Participants were classified as 'Inactive' (0 min/week of MVPA), 'Insufficiently Active (Low)' ( $<75 \mathrm{~min} /$ week of moderate-intensity PA or equivalent combination of MVPA), 'Insufficiently Active (High)' (75 to < 150 min/week of moderateintensity PA or equivalent combination of MVPA), or 'Sufficiently Active' ( $\geq 150 \mathrm{~min} /$ week of moderate-intensity PA or equivalent combination of MVPA) 


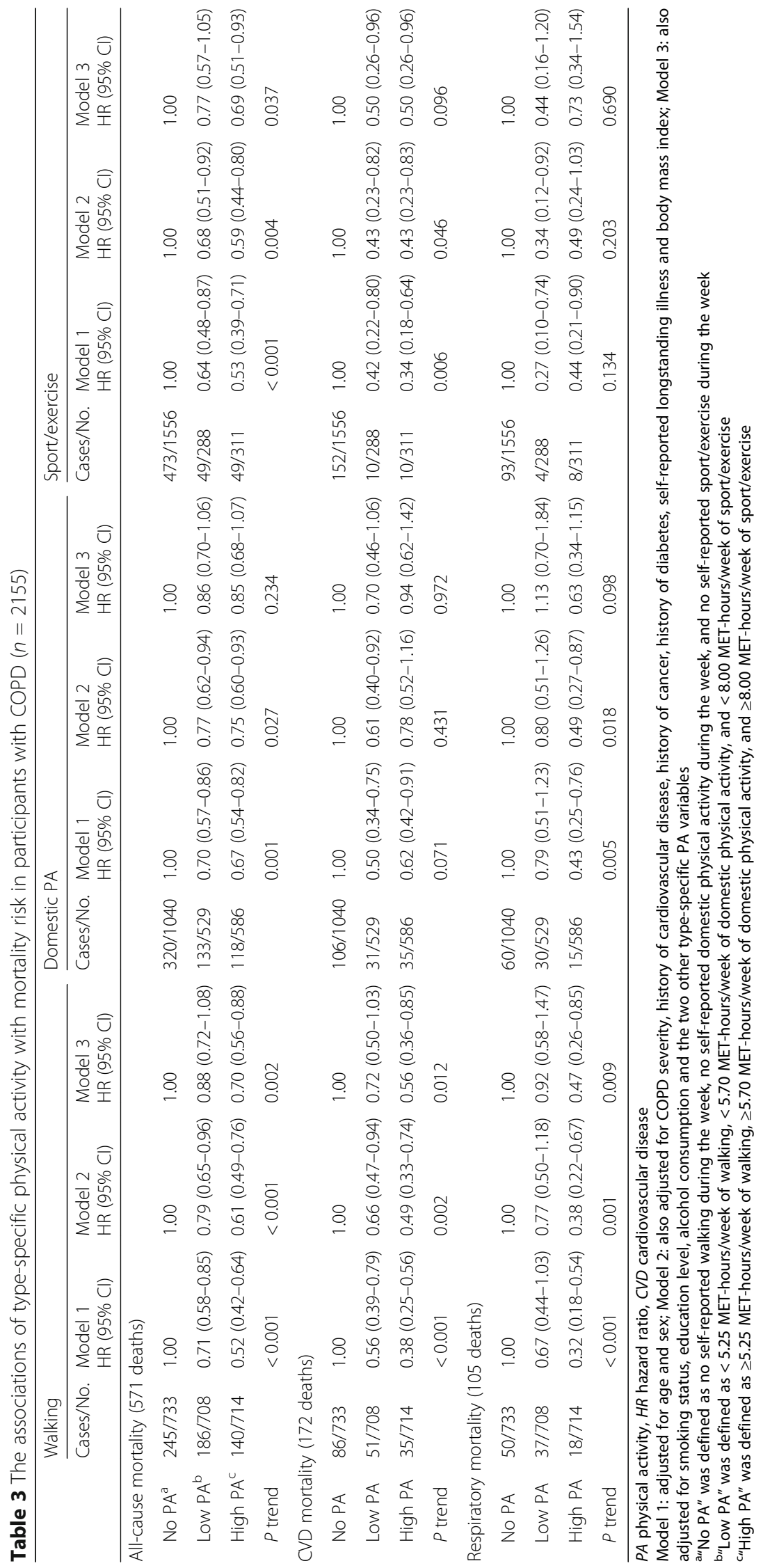


increasing MVPA in people with COPD, encouraging more light-intensity PA in this population may be a first and more realistic step than targeting MVPA alone.

Although there was a dose-response association between sport/exercise and mortality risk, sport is not feasible for most people with COPD due to the older age of the population and the high prevalence of comorbidities such as poly-pharmacy and mobility problems [21], which are associated with poorer physical performance [22]. Walking, however, is an achievable and accessible type of PA. It requires no specialised exercise equipment and, in the form of ground walking or treadmill walking, is commonly undertaken as part of pulmonary rehabilitation programs with good effect [23]. This study showed that participants who reported at least 5.25 MET-hours/week of walking (approximately equivalent to $105 \mathrm{~min} /$ week of brisk walking) had a $30 \%$ reduction in all-cause mortality risk, a $44 \%$ reduction in CVD mortality risk, and a 53\% reduction in respiratory mortality risk compared to those who reported no walking. Future studies should explore strategies for incorporating more walking into daily life for people with COPD. Nordic walking is one such strategy, where improvements in PA were maintained to 3 months in the group of participants [24].

Although domestic PA is feasible for most people with COPD, this study showed no association between domestic PA of any level and mortality risk. There is conflicting evidence about the protective effect of domestic PA on mortality. The domain is often poorly defined; for example, housework and gardening are both treated as domestic PA despite most housework activities being classified as light intensity, since they do not require locomotion or the use of large muscles groups [25]. An English population-based study of 15,000 adults found that PA "around the home", such as lawn mowing and digging, was inversely related to all-cause mortality risk [26]. However, a recent study of the SHS cohort found no association between intense domestic PA and CVD mortality risk after adjusting for relevant confounders [17]. The inclusion of more light intensity activities in our definition of domestic PA may explain the absence of a dose-response association between domestic PA and mortality risk.

Our study has several limitations. A fixed $\mathrm{FEV}_{1} / \mathrm{FVC}$ ratio was used to identify participants with COPD due to a lack of other information that may have helped to confirm a diagnosis. Although COPD can be identified with high diagnostic accuracy using spirometry, a fixed ratio of obstruction would not take into account any reversible factors that may have influenced $\mathrm{FEV}_{1}$, such as asthma or a respiratory infection on the day of testing. Spirometry performed on 219 adults in a general practice setting showed a sensitivity of $0.92(95 \%$ CI $0.80-0.97)$ for diagnosing COPD, with eight "false positives" identified from further laboratory testing [27]. To account for misclassification of COPD in our study, we conducted sensitivity analyses which excluded participants with asthma and/or a recent respiratory infection, and these yielded similar results to the main analysis.

Misclassification of the PA variables is also possible, since self-reported methods were used to quantify PA and any changes in PA levels during follow-up were not accounted for. This would have most likely reduced the magnitude of the effect of PA on mortality risk [28]. It is well-established that self-report PA questionnaires tend to overestimate the time spent physically active [29] as they are reliant on respondent recall and are often subject to social desirability bias [30], and thus should be interpreted with caution. Nevertheless, self-report questionnaires are frequently used to estimate PA levels in national populations for reasons of feasibility, cost-effectiveness, and to obtain information on specific types and domains of PA, which are not captured by objective measures. In comparison with accelerometry, the PASBAQ has demonstrated criterion validity similar to that of other questionnaires used in national surveys [15]. While there is no universally acceptable level for the magnitude of criterion validity coefficients for self-report PA questionnaires versus accelerometry, multiple reviews have indicated that correlations rarely exceed 0.40 [31-33].

Smoking history was not used as an eligibility criterion to identify participants with COPD due to a lack of available data to quantify smoking history in pack-years, and to preserve sample size. While the high prevalence of never-smokers in our sample (27.9\%) is surprising, it is supported by a recent case-finding study of 74,818 patients with COPD showing that $20-26 \%$ of patients were never-smokers [34]. Exposure to second-hand smoke, pollutants, and occupational exposure to dusts and chemicals are other risk factors for COPD [3], and may explain the high proportion of never-smokers in the sample. Furthermore, sensitivity analyses excluding never-smokers yielded similar results to the main analysis. It is therefore unlikely that the inclusion of never-smokers in the analysis would have biased the magnitude of the effect of PA on mortality risk.

Finally, given that the HSE and SHS are general population-based health examinations and are not specifically designed to investigate people with COPD, some potential confounders may not have been captured and this may limit the generalisability of the results. These include history of previous exacerbations, COPD hospitalisations, exercise capacity at baseline, and severity of symptoms, which are known predictors of mortality [35-37]. The high proportion of mild and moderate COPD in the sample may also limit the generalisability of the results to people with COPD of greater severity. 


\section{Conclusions}

We observed a linear dose-response association between PA and all-cause, CVD and respiratory mortality risk in people with COPD. There is added mortality benefit in adhering to the PA guidelines, although engaging in PA above a threshold of $3.75 \mathrm{MET}$-hours/week (at least $75 \mathrm{~min} /$ week of MPA, $32.5 \mathrm{~min}$ of VPA, or an equivalent combination of MVPA) is still associated with significant reductions in mortality risk. Walking and structured exercise should be encouraged in people with COPD, but the effects of domestic PA on mortality remain unclear. Our results reinforce the importance of promoting regular PA as part of routine management of COPD, and the need for effective strategies to increase PA in this population.

\section{Additional file}

Additional file 1: Tables S1-S4. The results of the sensitivity analyses are shown in the following supplementary tables. Table $\mathrm{S} 1$ shows the associations of total physical activity and moderate-vigorous intensity physical activity with mortality risk in participants with COPD without existing cardiovascular disease, cancer and diabetes at baseline. Table S2. shows the associations of total physical activity and moderate-vigorous intensity physical activity with mortality risk in participants with COPD with a smoking history but no coexisting asthma or recent respiratory infection. Table S3. shows the associations of type-specific physical activity with mortality risk in participants with COPD without existing cardiovascular disease, cancer and diabetes at baseline. Table S4. shows the associations of type-specific physical activity with mortality risk in participants with COPD with a smoking history but no coexisting asthma or recent respiratory infection. (DOCX $47 \mathrm{~kb}$ )

\section{Abbreviations \\ ANOVA: Analysis of variance; BMI: Body mass index; Cl: Confidence interval; COPD: Chronic obstructive pulmonary disease; CVD: Cardiovascular disease; $\mathrm{FEV}_{1}$ : Forced expiratory volume in one second; FVC: Forced vital capacity; GOLD: Global Initiative for Chronic Obstructive Lung Disease; HR: Hazard ratio; HSE: Health Survey for England; ICD: International Classification of Diseases; MET: Metabolic equivalent; MPA: Moderate-intensity physical activity; MVPA: Moderate-vigorous intensity physical activity; PA: Physical activity; PASBAQ: Physical Activity and Sedentary Behaviour Assessment Questionnaire; RR: Risk ratio; SHS: Scottish Health Survey; VMU: Vector- magnitude unit; VPA: Vigorous-intensity physical activity}

\section{Acknowledgements}

We would like to thank the study participants for their time.

\section{Funding}

ES is funded by the National Institute for Health Research (UK) (award code: CDF2010-03-30) and the National Health and Medical Research Council (award code: APP1110526) for dataset acquisition, processing and harmonisation.

\section{Availability of data and materials}

Mortality data from the Health Survey for England and Scottish Health Survey datasets that were analysed during the current study are not publicly available due to the risk of compromising individual privacy, but are available from the corresponding author on reasonable request.

\section{Authors' contributions}

SC, ZM, JA, SD and ES participated in the design of the study; SC performed the statistical analyses, and drafted and revised the paper; and all the authors contributed to the interpretation of results and revision of the draft paper. ES and $\mathrm{MH}$ were responsible for dataset acquisition, processing and harmonisation. ES had full access to the data, and SC had partial controlled access in a closed secure data environment; they both take joint responsibility for the integrity of the data and the accuracy of the data analyses. All authors read and approved the final manuscript.

\section{Ethics approval and consent to participate}

Ethics approvals for the Health Survey for England and Scottish Health Survey were obtained from the London Research Ethics Council and Scotland Local Research Ethics Councils respectively. Participants gave written informed consent.

\section{Consent for publication}

Not applicable.

\section{Competing interests}

The authors declare that they have no competing interests.

\section{Publisher's Note}

Springer Nature remains neutral with regard to jurisdictional claims in published maps and institutional affiliations.

\section{Author details}

${ }^{1}$ Discipline of Physiotherapy, The University of Sydney, Sydney, Australia. ${ }^{2}$ Ingham Institute for Applied Medical Research, Sydney, Australia. ${ }^{3}$ University of Loughborough, Loughborough, UK. ${ }^{4}$ Charles Perkins Centre, School of Public Health, Prevention Research Collaboration, The University of Sydney, Sydney, Australia.

Received: 26 September 2017 Accepted: 8 February 2018

Published online: 17 February 2018

\section{References}

1. Pitta F, Troosters T, Spruit MA, Probst VS, Decramer M, Gosselink R. Characteristics of physical activities in daily life in chronic obstructive pulmonary disease. Am J Respir Crit Care Med. 2005;171(9):972-7.

2. Vorrink SN, Kort HS, Troosters T, Lammers JW. Level of daily physical activity in individuals with COPD compared with healthy controls. Respir Res. 2011; 12:33. https://doi.org/10.1186/1465-9921-12-33.

3. Vogelmeier CF, Criner GJ, Martinez FJ, et al. Global strategy for the diagnosis, management, and prevention of chronic obstructive lung disease 2017 report. GOLD executive summary. Am J Respir Crit Care Med. 2017; 195(5):557-82.

4. Gimeno-Santos E, Frei A, Steurer-Stey C, et al. Determinants and outcomes of physical activity in patients with COPD: a systematic review. Thorax. 2014; 69(8):731-9.

5. Moy ML, Gould MK, Liu IA, Lee JS, Nguyen HQ. Physical activity assessed in routine care predicts mortality after a COPD hospitalisation. ERJ Open Res. 2016;2(1) https://doi.org/10.1183/23120541.00062-2015.

6. Watz $\mathrm{H}$, Pitta F, Rochester $\mathrm{CL}$, et al. An official European Respiratory Society statement on physical activity in COPD. Eur Respir J. 2014;44(6):1521-37.

7. Esteban C, Quintana JM, Aburto M, Moraza J, Capelastegui A. A simple score for assessing stable chronic obstructive pulmonary disease. QJM. 2006; 99(11):751-9.

8. Garcia-Rio F, Rojo B, Casitas R, et al. Prognostic value of the objective measurement of daily physical activity in patients with COPD. Chest. 2012;142(2):338-46.

9. Pitta F, Troosters T, Probst VS, Spruit MA, Decramer M, Gosselink R. Quantifying physical activity in daily life with questionnaires and motion sensors in COPD. Eur Respir J. 2006;27(5):1040-55.

10. World Health Organisation. Global strategy on diet, physical activity and health. Geneva: World Health Organization; 2010. Available from: http:// www.who.int/dietphysicalactivity/factsheet_adults/en/. Cited 1 Jul 2017

11. Mindell J, Biddulph JP, Hirani V, et al. Cohort profile: the health survey for England. Int J Epidemiol. 2012;41(6):1585-93.

12. Gray L, Batty GD, Craig P, et al. Cohort profile: the Scottish health surveys cohort: linkage of study participants to routinely collected records for mortality, hospital discharge, cancer and offspring birth characteristics in three nationwide studies. Int J Epidemiol. 2010;39(2):345-50.

13. Mindell J, Moody A, Bromley C, Rutherford L. The Scottish health survey: lung function in adults: topic report. Edinburgh: Produced for the Scottish 
Government by APS Group Scotland; 2014. Available from: www.gov.scot/ Resource/0045/00456687.pdf. Cited 1 Jul 2017

14. Ainsworth BE, Haskell WL, Herrmann SD, et al. Compendium of physical activities: a second update of codes and MET values. Med Sci Sports Exerc 2011;43(8):1575-81.

15. Scholes S, Coombs N, Pedisic Z, et al. Age- and sex-specific criterion validity of the health survey for England physical activity and sedentary behavior assessment questionnaire as compared with accelerometry. Am J Epidemiol. 2014;179(12):1493-502.

16. Arem H, Moore SC, Patel A, et al. Leisure time physical activity and mortality: a detailed pooled analysis of the dose-response relationship. JAMA Intern Med. 2015;175(6):959-67.

17. Stamatakis E, Hamer M, Lawlor DA. Physical activity, mortality, and cardiovascular disease: is domestic physical activity beneficial? The Scottish Health Survey - 1995, 1998, and 2003. Am J Epidemiol. 2009; 169(10):1191-200

18. Garcia-Aymerich J, Lange P, Benet M, Schnohr P, Antó JM. Regular physical activity reduces hospital admission and mortality in chronic obstructive pulmonary disease: a population based cohort study. Thorax. 2006;61(9): $772-8$.

19. Donaire-Gonzalez D, Gimeno-Santos E, Balcells E, et al. Benefits of physical activity on COPD hospitalisation depend on intensity. Eur Respir J. 2015; 46(5):1281-9.

20. Park SK, Larson JL. The relationship between physical activity and metabolic syndrome in people with chronic obstructive pulmonary disease. J Cardiovasc Nurs. 2014;29(6):499-507.

21. Schnell K, Weiss CO, Lee T, Krishnan JA, Leff B, Wolff JL, Boyd C. The prevalence of clinically-relevant comorbid conditions in patients with physician-diagnosed COPD: a cross-sectional study using data from NHANES 1999-2008. BMC Pulm Med. 2012;12:26. https://doi.org/10.1186/ 1471-2466-12-26.

22. Li LS, Caughey GE, Johnston KN. The association between co-morbidities and physical performance in people with chronic obstructive pulmonary disease: a systematic review. Chron Respir Dis. 2014;11(1):3-13.

23. Wootton SL, Ng LWC, McKeough ZJ, et al. Ground-based walking training improves quality of life and exercise capacity in COPD. Eur Respir J. 2014; 44(4):885-94.

24. Breyer MK, Breyer-Kohansal R, Funk GC, et al. Nordic walking improves daily physical activities in COPD: a randomised controlled trial. Respir Res. 2010; 11:112. https://doi.org/10.1186/1465-9921-11-112.

25. Rosano A, Moccaldi R, Cioppa M, Lanzieri G, Persechino B, Spagnolo A. Musculoskeletal disorders and housework in Italy. Ann Ig. 2004;16(3): 497-507.

26. Besson $H$, Ekelund $U$, Brage $S$, et al. Relationship between subdomains of total physical activity and mortality. Med Sci Sports Exerc. 2008; 40(11):1909-15.

27. Schneider $A$, Gindner $L$, Tilemann $L$, et al. Diagnostic accuracy of spirometry in primary care. BMC Pulm Med. 2009;9:31. https://doi.org/10.1186/14712466-9-31.

28. Andersen LB. Relative risk of mortality in the physically inactive is underestimated because of real changes in exposure level during follow-up. Am J Epidemiol. 2004;160(2):189-95.

29. Celis-Morales CA, Perez-Bravo F, Ibañez L, Salas C, Bailey M, Gill J. Objectiveversus-self-reported physical activity and sedentary time: effects of measurement method on relationships with risk biomarkers. PLoS One. 2012;7(5):e36345.

30. Adams SA, Matthews CE, Ebbeling CB, et al. The effect of social desirability and social approval on self-reports of physical activity. Am J Epidemiol. 2005;161(4):389-98.

31. Bauman A, Phongsavan P, Schoeppe S, Owen N. Physical activity measurement-a primer for health promotion. Promot Educ. 2006; 13(2):92-103.

32. Prince SA, Adamo KB, Hamel ME, Hardt J, Connor Gorber S, Tremblay M. A comparison of direct versus self-report measures for assessing physical activity in adults: a systematic review. Int J Behav Nutr Phys Act. 2008;5:56. https://doi.org/10.1186/1479-5868-5-56.

33. Shephard RJ. Limits to the measurement of habitual physical activity by questionnaires. Br J Sports Med. 2003;37(3):197-206.

34. Jordan RE, Adab P, Sitch A, et al. Targeted case finding for chronic obstructive pulmonary disease versus routine practice in primary care
(TargetCOPD): a cluster-randomised controlled trial. Lancet Respir Med. 2016:4(9):720-30.

35. Almagro P, Calbo E, Ochoa de Echagüen A, et al. Mortality after hospitalization for COPD. Chest. 2002;121(5):1441-8.

36. Garcia-Aymerich J, Monsó E, Marrades RM, et al. Risk factors for hospitalization for a chronic obstructive pulmonary disease exacerbation: EFRAM study. Am J Respir Crit Care Med. 2001;164(6):1002-7.

37. McGhan R, Radcliffe T, Fish R, Sutherland ER, Welsh C, Make B. Predictors of rehospitalization and death after a severe exacerbation of COPD. Chest. 2007;132(6):1748-55.

\section{Submit your next manuscript to BioMed Central and we will help you at every step:}

- We accept pre-submission inquiries

- Our selector tool helps you to find the most relevant journal

- We provide round the clock customer support

- Convenient online submission

- Thorough peer review

- Inclusion in PubMed and all major indexing services

- Maximum visibility for your research

Submit your manuscript at www.biomedcentral.com/submit
Biomed Central 